\title{
Coronary Artery Spasm is a Nightmare: A Rare Case of Multi Vessel Coronary Artery Spasm
}

\section{Wang $J Y^{*}$, Chen $\mathrm{H}$ and Su $\mathrm{X}$}

Department of Cardiology, Wuhan Asia Heart Hospital, Wuhan, PR China

\begin{abstract}
Coronary artery spasm is not an uncommon event, but its pathophysiologic mechanisms followed by coronary artery vasospasm are not yet completely clarified. Coronary artery spasm can lead to sudden cardiac death due to ventricular arrhythmias or heart block. In the cases of cardiogenic shock secondly to severe coronary artery spasm, IABP is sufficient to support the patient's hemodynamics, in the other cases of severe cardiogenic shock or cardiac arrest secondly to severe coronary artery spasm, IABP is not sufficient to support the patient's hemodynamics and more advanced mechanical circulatory support devices such as veno-arterial ECMO should be performed. This report describes a case of severe multi vessel coronary artery spasm leading to cardiac arrest, cardiogenic shock and cardiac death although using the first-class heart circulation assist device, and illustrates IABP and ECMO support for acute cardiac dysfunction secondary to diffuse multivessel coronary artery spasm was fruitless labour sometimes.
\end{abstract}

Keywords: Coronary artery spasm; Cardiac arrest; Intra-aortic balloon pump; Extracorporeal membrane oxygenation

\section{Introduction}

Coronary artery spasm is not an uncommon event, but its pathophysiologic mechanisms followed by coronary artery vasospasm are not yet completely clarified. Coronary endothelial dysfunction and a variety of nonspecific vasoconstrictive stimuli factors such as $\alpha$-adrenergic stimulation, $\beta$-adrenergic blockade, and altered sympathovagal balance could be the main causes of coronary artery spasm $[1,2]$. Patients with coronary artery spasm typically describe chest pain symptoms, usually occurring in the early hours of the morning with ST-segment elevation on ECG. In severe cases, such as multivessel coronary artery spasm associated arrhythmias may be present ranging from heart block to ventricular tachycardia [3].

\section{Case Report}

A 64 years' old male was admitted to our heart-centre complaining of chest distress and inhalation for fifteen days. His initial vital signs included temperature $36.6^{\circ} \mathrm{C}$, heart rate 78 beats $/ \mathrm{min}$, blood pressure $106 / 60 \mathrm{mmHg}$, and respiratory rate $20 \mathrm{breaths} / \mathrm{min}$, jugular vein distension, diastolic mild sigh murmur heard at the first and second auscultation area of aortic valve without conduction, systolic blowing murmur heard at heart apex with left armpit conduction, hepatojugular reflux sign positive. ECG revealed atrial fibrillation arrhythmia without change of ST segment or T wave. And chest X-ray had no acute changes. Laboratory examination revealed a leukocytosis of $7.68 \times 10^{9} / \mathrm{L}$, C-reactive protein of $3.02 \mathrm{mg} / \mathrm{L}$, and serum levels of troponin I is of $0.023 \mathrm{ng} / \mathrm{mL}$ (normal range is from 0 to $0.04 \mathrm{ng} / \mathrm{mL}$ ). After transthoracic echocardiography and other related inspection, $\mathrm{He}$ was diagnosed as "Heart Valve Disease: Moderate aortic valve insufficiency, severe mitral valve insufficiency, mild tricuspid valve reflux, atrial fibrillation, cardiac function grade III (NYHA class) “. Coronary angiography via the right radial artery demonstrated that atheromatous plaque invasion of the left anterior descending coronary artery (LAD) and right coronary artery (RCA), borderline lesions (the degree of stenosis $60 \%$ ) of the middle left circumflex coronary artery (LCX). He was treated by cardiac surgery under hypothermia and extracorporeal circulation: Aortic valve replacement (AVR, St. Jude 25\#) + Tricuspid valvuloplasty (TVP)
+ Minimally invasive bipolar radiofrequency ablation of lone atrial fibrillation (MAZE) + epicardium temporary pacemaker placement within 4 hours, and then transferred to the intensive care unit (ICU) administered conventional hemodynamic monitoring, continuous anesthesia, mechanical ventilation and supported by vasoactive drugs (ten points) and expectant treatment. Review of echocardiographic: no regurgitation of any valve after operation, left ventricular ejection fraction $45 \%$. Postoperative pathologic examination had shown that mitral and aortic valve featured by senile degenerative changes. Postoperation ECG revealed sinus rhythm without change of ST segment or T wave.

The tenth day after surgery at general ward, he experienced episode cardiac arrest, fall down at the toilet and then quickly was administered continuous cardio-pulmonary resuscitation (CPR), cardiac defibrillation, tracheal intubation and mechanical ventilation, the rhythm of the heart resumed sinus rhythm. After successful cardiopulmonary resuscitation, 12-lead ECG showed ST segment significantly elevation in the I, II, III, aVF, AVL and V1 to V6 leads, and occasional premature ventricular complexes, transthoracic echocardiography revealed severely global ventricle wall hypo kinesis and left ventricular ejection fraction of $15 \%$. Emergency coronary angiography revealed that the entire coronary artery systems diffuse spasm (Figures 1A and 2A). The patient's hemodynamic instability had not been adjusted despite administration of intracoronary boluses of nitroglycerin and diltiazem, intravenous vasopressors and intraaortic balloon pump (IABP) counter pulsation. Coronary angiography

*Corresponding author: Wang JY, Department of Cardiology, Wuhan Asia Heart Hospital, Wuhan 430022, PR China, Tel: +8615172496706; E-mail: drwangjiangyou@163.com

Received February 03, 2016; Accepted February 18, 2016; Published February 26, 2016

Citation: Wang JY, Chen H, Su X (2016) Coronary Artery Spasm is a Nightmare: A Rare Case of Multi Vessel Coronary Artery Spasm. J Vasc Med Surg 4: 254 doi:10.4172/2329-6925.1000254

Copyright: ( 2016 Wang JY, et al. This is an open-access article distributed unde the terms of the Creative Commons Attribution License, which permits unrestricted use, distribution, and reproduction in any medium, provided the original author and source are credited. 


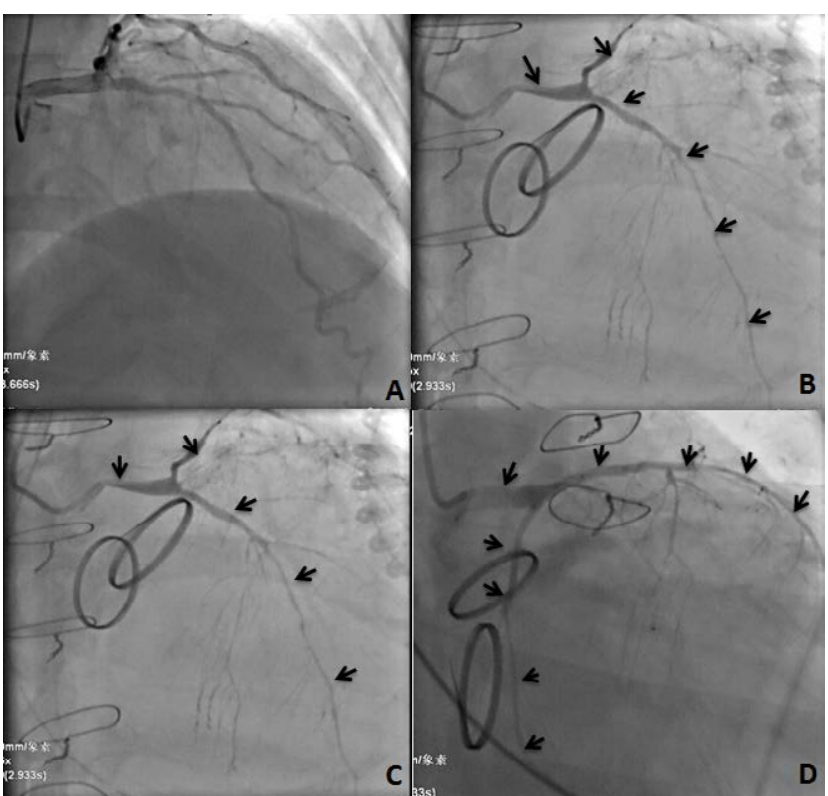

Figure 1: A) Coronary angiography image before operation revealed no abnormality at the left main artery, atheromatous plaque invasion (the degree of stenosis $20-40 \%$ ) of the LAD, borderline lesions (the degree of stenosis $60 \%$ ) of the middle LCX. B) Coronary angiography image after operation revealed the left main artery spasm (the degree of stenosis $80 \%$ indicated by the arrows), the LAD diffuse spasm (the degree of stenosis $95 \%$ indicated by the arrows), and the LCX diffuse spasm (the degree of stenosis $99 \%$ indicated by the arrows). C) Coronary angiography image after intravascular injection of $400 \mu \mathrm{g}$ nitroglycerin, left coronary vascular spasm had no obvious improvement indicated by the arrows. D) Coronary angiography image after repeated intravascular injection of $400 \mu \mathrm{g}$ nitroglycerin, left main artery spasm had been alleviated indicated by the arrow, but no improvement in LCX and LAD indicated by the arrows.

reassessment revealed no improvement of the entire coronary artery systems (Figures 1B-1D, 2B and 2C). IABP was emergently evacuated and replaced by extracorporeal membrane oxygenation (Veno-arterial ECMO) for hemodynamic stability. Under the ECMO support, the patient's hemodynamics was barely able to maintain. Laboratories revealed a leukocytosis of $24.43 \times 109 / \mathrm{L}$, creatinine of $198 \mu \mathrm{mol} /$ $\mathrm{L}$, blood urea nitrogen of $11.03 \mathrm{mmol} / \mathrm{l}$, glomerular filtration rate of $31 \mathrm{~mL} / \mathrm{min}$; total bilirubin of $45.4 \mu \mathrm{mol} / \mathrm{L}$, direct bilirubin of $30.0 \mu \mathrm{mol} / \mathrm{L}$, alb content of $18.7 \mathrm{~g} / \mathrm{L}$, troponin I of $2253.86 \mathrm{ng} / \mathrm{mL}$, $\mathrm{N}$-terminal pro brain natriuretic peptide of $4394 \mathrm{pg} / \mathrm{mL}$, lactate of $>15 \mathrm{mmol} / \mathrm{L}$, then continuously blood purifications was implemented. Sixteen hours later, the patient's blood pressure progressively dropped to $50 / 24 \mathrm{mmHg}$ with heart beats slowed down gradually, and then adrenaline and atropine intravenous injection repeatedly, circulation still could not restore. The ECG demonstrated electrical mechanical dissociation, mydriasis, various physiological reflexes were disappeared, and announced clinical death. ECMO lasted for 18 hours totally.

\section{Discussion}

Coronary artery spasm is not an uncommon event, but its pathophysiologic mechanisms followed by coronary artery vasospasm are not yet completely clarified. Coronary endothelial dysfunction and a variety of nonspecific vasoconstrictive stimuli factors such as $\boldsymbol{\alpha}$-adrenergic stimulation, $\boldsymbol{\beta}$-adrenergic blockade, and altered sympathovagal balance could be the main causes of coronary artery spasm $[1,2]$.Patients with coronary artery spasm typically describe chest pain symptoms, usually occurring in the early hours of the morning with ST-segment elevation on ECG. In severe cases, such as multivessel coronary artery spasm associated arrhythmias may be present ranging from heart block to ventricular tachycardia [3]. If it had not promptly diagnosed and treated, coronary artery spasm might result in myocardial infarction with life-threatening consequences. Clinically, coronary artery spasm may be discovered through the change in ECG, hemodynamic instability, arrhythmia, circulatory collapse, or cardiac arrest. Echocardiography may show new segmental wall motion abnormalities. However, the exact diagnosis of coronary artery spasm can be achieved only by coronary angiography. In our case, the patient experienced cardiac arrest followed with ST segment significantly elevation in the I, II, III, aVF, AVL and V1 to V6 leads, suggestive of extensive anterior myocardial and inferior wall myocardial infarction. The diffuse myocardial infarction caused malignant arrhythmia and cardiac arrest. In the process of cardiopulmonary resuscitation, using large dose of vasoactive drugs might deteriorate coronary artery spasm.

Multivessel coronary artery diffuse spasm can develop into myocardial ischemia, myocardial infarction, and cardiac dysfunction. Akashi et al. suggested that epinephrine can lead to multi-vessel epicardial or micro-vascular vasospasm and myocardial ischemia, which results in myocardial stunning and global ventricular dysfunction [4]. Once continuous hemodynamic deterioration secondary to coronary artery spasm is detected, treatment is extremely difficult. The vasoconstrictive and positive inotropic drugs will aggravate coronary artery spasm. On the other hand, the administration of intravenous coronary vasodilators such as nitroglycerin and calcium antagonists may exacerbate hemodynamic deterioration when complicated with cardiogenic shock. In this case, under the IABP support, the patient's hemodynamics was unimproved with administration of intravenous diltiazem and nitroglycerin. IABP was emergently replaced by ECMO for hemodynamic stability. Under the ECMO support, the

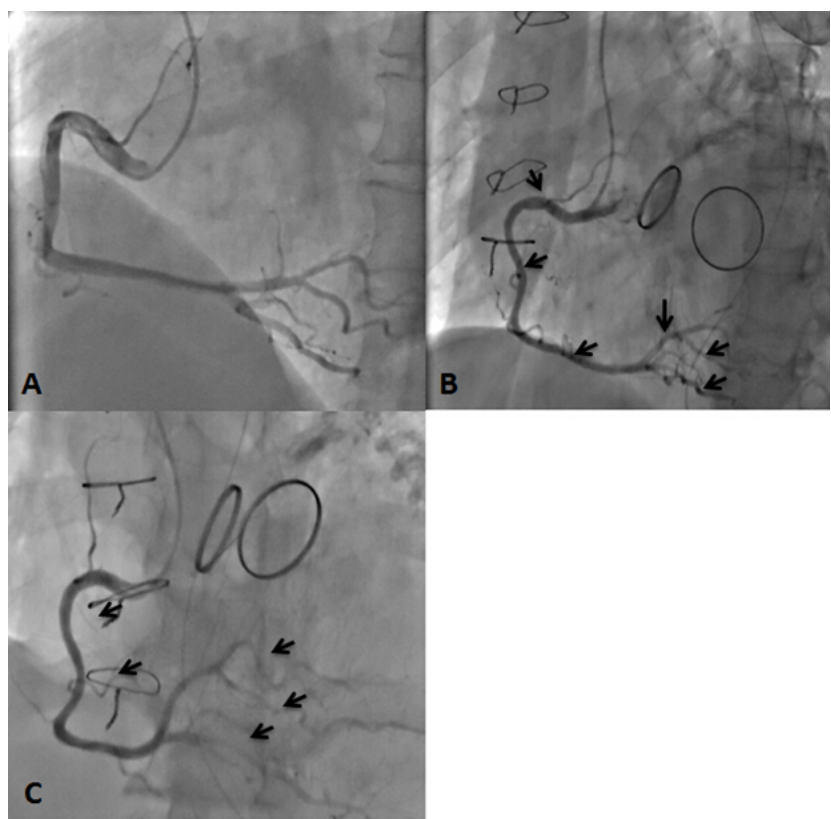

Figure 2: A) Coronary angiography image before operation revealed athermanous plaque invasion (the degree of stenosis $10-20 \%$ ) of the RCA. B) Coronary angiography image after operation revealed the RCA diffuse spasm (the degree of stenosis $60 \%$ indicated by the arrows). C) Coronary angiography image after intravascular injection of $400 \mu \mathrm{g}$ nitroglycerin, right coronary vascular spasm had no obvious improvement indicated by the arrows. 
patient's hemodynamics was barely able to maintain. He died from various complications. In the cases of cardiogenic shock or cardiac arrest secondly to severe coronary artery spasm, IABP is sufficient to support the patient's hemodynamics [5], in the other cases of severe cardiogenic shock or cardiac arrest secondly to severe coronary artery spasm, IABP is not sufficient to support the patient's hemodynamics and more advanced mechanical circulatory support devices such as veno-arterial ECMO should be performed [6]. After initiation of ECMO, catecholamine infusions which may aggravate coronary artery spasm by the alpha-adrenergic effect can be discontinued, since ECMO can provide full circulatory support. In addition, ECMO will improve oxygenation, decompress the heart and reduce myocardial oxygen consumption, therefore reducing any on-going ischemia. Once stabilized on ECMO, the patient can be safely transported to the catheterization laboratory for further evaluation. Support with ECMO combined with continuous use of intravenous vasodilators for spasm could be lifesaving in severe coronary artery spasm cases. Sadly, in this case, the patient's hemodynamics was unimproved with IABP or ECMO support.

Our case is an unusual to present multivessel coronary artery diffuse spasm with sudden cardiac arrest due to ventricular fibrillation. Sometimes, we should not underestimate the risk of coronary artery spasm. Coronary artery spasm always should be kept in mind because it can lead to serious life threatening complications. In the cases of severe cardiogenic shock or cardiac arrest secondary to coronary artery spasm, ECMO was also in vain.

\section{References}

1. Hirabayashi Y, Saitoh K, Fukuda H, Mitsuhata H, Shimizu R (1996) Coronary artery spasm after ephedrine in a patient with high spinal anesthesia. Anesthesiology 84: 221-224.

2. lida R, Yazaki S, Saeki S (2005) Recurrent ST-segment elevations in a patient without significant coronary disease. J Clin Anesth 17: 372-378.

3. Previtali M, Klersy C, Salerno JA, Chimienti M, Panciroli C, et al. (1983) Ventricular tachyarrhythmias in Prinzmetal's variant angina: clinical significance and relation to the degree and time course of S-T segment elevation. Am J Cardiol 52: 19-25.

4. Akashi YJ, Nakazawa K, Sakakibara M, Miyake F, Sasaka K (2002) Reversible left ventricular dysfunction "takotsubo" cardiomyopathy related to catecholamine cardiotoxicity. J Electrocardiol 35: 351-356.

5. Zhang Z, Su X, Liu C, Xu C, Wu M, et al. (2014) Utility of intra-aortic balloon pump support for multivessel coronary artery spasm and cardiac arrest. Int J Cardiol 176: e122-124.

6. Unai S, Hirose H, Cook G, Lee Y, Miura S, et al. (2014) Coronary artery spasm following off-pump coronary artery bypass surgery. Int Heart J 55: 451-454. 